\title{
Absolute stability of time-varying delay Lurie indirect control systems with unbounded coefficients
}

\author{
Fucheng Liao ${ }^{1 *}, X_{i a o} \mathrm{Yu}^{1}$ and Jiamei Deng ${ }^{2}$
}

\section{"Correspondence:}

fcliao@ustb.edu.cn

${ }^{1}$ School of Mathematics and

Physics, University of Science and

Technology Beijing, Beijing, 100083,

China

Full list of author information is

available at the end of the article

\begin{abstract}
This paper investigates the absolute stability problem of time-varying delay Lurie indirect control systems with variable coefficients. A positive-definite Lyapunov-Krasovskii functional is constructed. Some novel sufficient conditions for absolute stability of Lurie systems with single nonlinearity are obtained by estimating the negative upper bound on its total time derivative. Furthermore, the results are generalised to multiple nonlinearities. The derived criteria are especially suitable for time-varying delay Lurie indirect control systems with unbounded coefficients. The effectiveness of the proposed results is illustrated using simulation examples.
\end{abstract}

Keywords: nonlinear systems; Lurie indirect control systems; absolute stability; Lyapunov stability theorem

\section{Introduction}

In the middle of the last century, the concept of absolute stability was introduced in [1]. Since then, the absolute stability problem of Lurie system has been extensively studied in the academic community, and there have been many publications on this topic [2-6]. As for time-delay Lurie systems with constant coefficients, fruitful results have been obtained. In [7], Khusainov and Shatyrko studied the absolute stability of multi-delay regulation systems. In [8], by applying the properties of $M$-matrix and selecting an appropriate Lyapunov function, Chen et al. established new absolute stability criteria for Lurie indirect control system with multiple variable delays, and they improved and generalised the corresponding results in [9]. In [10, 11], different Lyapunov-Krasovskii functionals were constructed. The absolute stability problem of Lurie direct control system with multiple time-delays became the stability problem of a neutral-type system based on the NewtonLeibniz formula and decomposing the matrices, and some stability criteria were obtained. The authors in $[12,13]$ made greater improvements. They avoided the stability assumption on the operator using extended Lyapunov functional and gave less conservative stability criteria than those in [10,11]. [14] and [15] studied the absolute stability of Lurie systems with constant delay and the systems with time-varying delay, respectively. Improved robust absolute stability criteria were obtained in [16] and [17] based on a free-weighting matrix approach and a delayed decomposition approach. Additionally, for a class of more complicated Lurie indirect control systems of neutral type, some relevant stability conditions were derived in [18-20].

(c) The Author(s) 2017. This article is distributed under the terms of the Creative Commons Attribution 4.0 International License (http://creativecommons.org/licenses/by/4.0/), which permits unrestricted use, distribution, and reproduction in any medium, provided you give appropriate credit to the original author(s) and the source, provide a link to the Creative Commons license, and indicate if changes were made. 
At the same time, Lurie system has been generalised by researchers from different aspects. Time-varying Lurie system is a natural generalisation. For the absolute stability of such a system, there have been lots of useful results. In [21], the absolute stability of Lurie indirect control systems and large-scale systems with multiple operators and unbounded coefficients were studied. The discussed system was taken as a large-scale interconnected system composed of several subsystems. By constructing a Lyapunov function for each isolated subsystem, a certain weighted sum of them was considered as the Lyapunov function of the original system. Thus some stability criteria were derived. The authors in [22, 23] developed some sufficient conditions for the absolute stability of Lurie direct control systems and large-scale systems with unbounded coefficients.

Regarding the absolute stability of time-varying Lurie systems, uncertain Lurie systems and stochastic Lurie systems, lots of research results have been reported in the literature. However, most of the results on the absolute stability of Lurie systems require that the system coefficients be bounded. Motivated by this, we will study the absolute stability of time-varying Lurie indirect control systems with time delay. Especially, the coefficients of the system studied in this paper can be unbounded. Lyapunov's second method will be used. In fact, the research methods in $[14,15,21]$ can be combined and modified appropriately to investigate the systems considered in this paper. The proposed LyapunovKrasovskii functional not only keeps the components related to a quadratic form together with an integral term in the above references, but also adds an integral of a quadratic form related to the time delay. Finally, several new simple absolute stability criteria are established. The novelty of the paper can be summarised as follows: The elements of the system coefficient matrices can be unbounded functions; and also the time delay can be very large if its time derivative is less than one. At the same time, the obtained results are also applicable to time-varying delay Lurie indirect control systems with bounded coefficients and the systems with constant coefficients.

Notation Throughout this paper, $\lambda(A)$ stands for any eigenvalue of the square matrix $A$; Let vector $x=\left[\begin{array}{llll}x_{1} & x_{2} & \cdots & x_{m}\end{array}\right]^{T}$, and $\|x\|$ represents the Euclidean norm of the vector $x$, i.e. , $\|x\|=\sqrt{\sum_{i=1}^{m} x_{i}^{2}}$; The matrix norm $\|A\|$, induced by the Euclidean vector norm $\|x\|$, is defined as $\|A\|=\max _{\|x\|=1}\|A x\|$, and it can be easily verified that $\|A\|=\sqrt{\lambda_{\max }\left(A^{T} A\right)}$; $\varlimsup_{t \rightarrow \infty}$ refers to the upper limit. For simplicity, let $\phi(\theta)=\left[\begin{array}{c}x(t+\theta) \\ \sigma(t)\end{array}\right], \theta \in[-h, 0], t \geq 0$, $\|\phi\|_{L_{2}}=\sqrt{\int_{-h}^{0}\|\phi(\theta)\|^{2} d \theta}$.

Lurie indirect control systems with single nonlinearity will be first studied, and then the derived results will be extended to multiple nonlinearities. Lyapunov's theorem on asymptotic stability of time-delay systems used in the proof is given in $[24,25]$. For the case of multiple nonlinearities, $\sigma(t)$ in $\phi(\theta)$ is taken as a vector.

\section{Absolute stability of Lurie systems with single nonlinearity}

Consider the following time-varying delay Lurie indirect control system with variable coefficients and single nonlinearity:

$$
\left\{\begin{array}{l}
\dot{x}(t)=A(t) x(t)+B(t) x(t-\tau(t))+b(t) f(\sigma(t)), \\
\dot{\sigma}(t)=c^{T}(t) x(t)-\rho(t) f(\sigma(t)), \\
x(t)=\varphi(t), \quad t \in[-h, 0]
\end{array}\right.
$$


where $x(t) \in R^{n} ; \sigma(t) \in R ; A(t), B(t)$ are $n \times n$ matrices, $b(t), c(t)$ are $n$-dimensional column vectors; $\tau(t)$ is time delay; $\rho(t) \geq \rho>0, \rho$ is a constant. $A(t), B(t), b(t), c(t), \rho(t)$ are continuous in $[0, \infty), \varphi(t)$ is the initial condition. The nonlinearity $f(\cdot)$ is continuous and satisfies the sector condition:

$$
F_{\left[k_{1}, k_{2}\right]}=\left\{f(\cdot) \mid f(0)=0 ; k_{1} \sigma^{2}(t) \leq \sigma(t) f(\sigma(t)) \leq k_{2} \sigma^{2}(t), \sigma(t) \in R-\{0\}\right\},
$$

where $k_{1}, k_{2}$ are given constants satisfying $k_{2}>k_{1}>0$.

Definition 1 ([26]) System (1) is said to be absolutely stable if its zero solution is globally asymptotically stable for any nonlinearity $f(\cdot) \in F_{\left[k_{1}, k_{2}\right]}$.

For system (1), the following assumptions are made.

A1: The time delay $\tau(t)$ denotes the continuous and piecewise differentiable function satisfying

$$
0 \leq \tau(t) \leq h, \quad \dot{\tau}(t) \leq \alpha<1,
$$

where $h, \alpha$ are constants. At the non-differential points of $\tau(t), \dot{\tau}(t)$ represents $\max [\dot{\tau}(t-0), \dot{\tau}(t+0)]$.

A2: For any $t \in[0, \infty)$, there exist symmetric positive-definite matrices $P$ and $G$ such that

$$
\lambda\left(P A(t)+A^{T}(t) P+G\right) \leq-\delta(t) \leq-\xi<0,
$$

where $\delta(t)>0$ is a function and $\xi>0$ is a constant.

A3: For any $t \in[0, \infty)$, assume that

$$
\frac{\|P B(t)\|}{\sqrt{\delta(t)(1-\alpha) \lambda_{\min }(G)}} \leq \eta, \quad \frac{\left\|P b(t)+\frac{1}{2} c(t)\right\|}{\sqrt{\delta(t) \rho(t)}} \leq \gamma,
$$

where $\eta, \gamma$ are constants.

Theorem 1 Under A1, A2 and A3, if the inequality

$$
\eta^{2}+\gamma^{2}<1
$$

holds, then system (1) is absolutely stable.

Proof Using the matrices $P$ and $G$, a Lyapunov-Krasovskii functional candidate is chosen as

$$
V(t, \phi)=x^{T}(t) P x(t)+\int_{t-\tau(t)}^{t} x^{T}(s) G x(s) d s+\int_{0}^{\sigma(t)} f(s) d s .
$$


It can be proved that if $f \in F_{\left[k_{1}, k_{2}\right]}$, then $\frac{1}{2} k_{1} \sigma^{2}(t) \leq \int_{0}^{\sigma(t)} f(s) d s \leq \frac{1}{2} k_{2} \sigma^{2}(t)$ hold. Thus, $V$ satisfies

$$
\begin{aligned}
& \lambda_{\min }(P)\|x(t)\|^{2}+\frac{1}{2} k_{1} \sigma^{2}(t) \\
& \leq V(t, \phi) \leq \lambda_{\max }(P)\|x(t)\|^{2}+\frac{1}{2} k_{2} \sigma^{2}(t)+\lambda_{\max }(G) \int_{-h}^{0}\|x(t+\theta)\|^{2} d \theta .
\end{aligned}
$$

Further, we have

$$
\begin{aligned}
& \min \left\{\lambda_{\min }(P), \frac{1}{2} k_{1}\right\}\|\phi(0)\|^{2} \\
& \leq V(t, \phi) \leq \max \left\{\lambda_{\max }(P), \frac{1}{2} k_{2}\right\}\|\phi(0)\|^{2}+\lambda_{\max }(G) \int_{-h}^{0}\|\phi(\theta)\|^{2} d \theta .
\end{aligned}
$$

That is, let

$$
u(s)=\min \left\{\lambda_{\min }(P), \frac{1}{2} k_{1}\right\} s^{2}, \quad v_{1}(s)=\max \left\{\lambda_{\max }(P), \frac{1}{2} k_{2}\right\} s^{2}, \quad v_{2}(s)=\lambda_{\max }(G) s^{2},
$$

then the following will hold when $t \geq 0$ :

$$
u(\|\phi(0)\|) \leq V(t, \phi) \leq v_{1}(\|\phi(0)\|)+v_{2}\left(\|\phi\|_{L_{2}}\right)
$$

Consequently, $V(t, \phi)$ satisfies the conditions required by Lyapunov's theorem.

The time derivative of $V(t, \phi)$ along the trajectories of system (1) will be calculated, and its upper bound will be estimated as follows:

$$
\begin{aligned}
\frac{d}{d t} V(t, \phi) & \\
= & 2 x^{T}(t) P \dot{x}(t)+x^{T}(t) G x(t)-(1-\dot{\tau}(t)) x^{T}(t-\tau(t)) G x(t-\tau(t))+f(\sigma(t)) \dot{\sigma}(t) \\
= & 2 x^{T}(t) P[A(t) x(t)+B(t) x(t-\tau(t))+b(t) f(\sigma(t))]+x^{T}(t) G x(t) \\
& -(1-\dot{\tau}(t)) x^{T}(t-\tau(t)) G x(t-\tau(t))+f(\sigma(t))\left(c^{T}(t) x(t)-\rho(t) f(\sigma(t))\right) \\
= & x^{T}(t)\left[P A(t)+A^{T}(t) P+G\right] x(t)+2 x^{T}(t) P B(t) x(t-\tau(t)) \\
& +2 x^{T}(t) P b(t) f(\sigma(t))-(1-\dot{\tau}(t)) x^{T}(t-\tau(t)) G x(t-\tau(t)) \\
& +f(\sigma(t)) c^{T}(t) x(t)-\rho(t) f^{2}(\sigma(t)) .
\end{aligned}
$$

By virtue of A1, A2 and the property of norm, the following will be obtained:

$$
\begin{aligned}
\frac{d}{d t} V(t, \phi) & \\
\leq & -\delta(t)\|x(t)\|^{2}+2\|P B(t)\|\|x(t)\|\|x(t-\tau(t))\| \\
& +2\left\|P b(t)+\frac{1}{2} c(t)\right\|\|x(t)\||f(\sigma(t))| \\
& -(1-\alpha) \lambda_{\min }(G)\|x(t-\tau(t))\|^{2}-\rho(t) f^{2}(\sigma(t)) .
\end{aligned}
$$


In order to make full use of A3 and the unbounded terms in the coefficients of system (1), take $\sqrt{\delta(t)}\|x(t)\|, \sqrt{(1-\alpha) \lambda_{\min }(G)}\|x(t-\tau(t))\|$ and $\sqrt{\rho(t)}|f(\sigma(t))|$ as the following variables of the quadratic form. By further estimating the right-hand side of $\left.\frac{d}{d t} V(t, \phi)\right|_{(1)}$ based on A3, let us note that

$$
\begin{aligned}
&\left.\frac{d}{d t} V(t, \phi)\right|_{(1)} \\
& \leq- \delta(t)\|x(t)\|^{2} \\
&+\frac{2\|P B(t)\|}{\sqrt{\delta(t)(1-\alpha) \lambda_{\min }(G)}}[\sqrt{\delta(t)}\|x(t)\|] \cdot\left[\sqrt{(1-\alpha) \lambda_{\min }(G)}\|x(t-\tau(t))\|\right] \\
&+2 \frac{\left\|P b(t)+\frac{1}{2} c(t)\right\|}{\sqrt{\delta(t) \rho(t)}}[\sqrt{\delta(t)}\|x(t)\|] \cdot[\sqrt{\rho(t)}|f(\sigma(t))|] \\
&-(1-\alpha) \lambda_{\min }(G)\|x(t-\tau(t))\|^{2}-\rho(t) f^{2}(\sigma(t)) \\
& \leq-\delta(t)\|x(t)\|^{2}+2 \eta[\sqrt{\delta(t)}\|x(t)\|] \cdot\left[\sqrt{(1-\alpha) \lambda_{\min }(G)}\|x(t-\tau(t))\|\right] \\
&+2 \gamma[\sqrt{\delta(t)}\|x(t)\|] \cdot[\sqrt{\rho(t)}|f(\sigma(t))|] \\
&-(1-\alpha) \lambda_{\min }(G)\|x(t-\tau(t))\|^{2}-\rho(t) f^{2}(\sigma(t)) .
\end{aligned}
$$

Then, rewriting the right-hand side of the above inequality yields

$$
\begin{aligned}
\left.\frac{d}{d t} V(t, \phi)\right|_{(1)} \leq & {\left[\begin{array}{c}
\sqrt{\delta(t)}\|x(t)\| \\
\sqrt{(1-\alpha) \lambda_{\min }(G)}\|x(t-\tau(t))\| \\
\sqrt{\rho(t)}|f(\sigma(t))|
\end{array}\right]^{T} } \\
& \times D\left[\begin{array}{c}
\sqrt{\delta(t)}\|x(t)\| \\
\sqrt{(1-\alpha) \lambda_{\min }(G)}\|x(t-\tau(t))\| \\
\sqrt{\rho(t)}|f(\sigma(t))|
\end{array}\right],
\end{aligned}
$$

where

$$
D=\left[\begin{array}{ccc}
-1 & \eta & \gamma \\
\eta & -1 & 0 \\
\gamma & 0 & -1
\end{array}\right] .
$$

In the following, we will show that the right-hand side of (2) is a negative-definite function. To establish this result, let us prove that matrix $D$ is negative definite. It is easy to obtain the characteristic polynomial of $D$ given by

$$
|\lambda I-D|=(\lambda+1)\left[(\lambda+1)^{2}-\left(\eta^{2}+\gamma^{2}\right)\right]
$$

Thus, the eigenvalues of $D$ are as follows:

$$
\lambda_{1}=-1, \quad \lambda_{2}=-1+\sqrt{\eta^{2}+\gamma^{2}}, \quad \lambda_{3}=-1-\sqrt{\eta^{2}+\gamma^{2}} .
$$


It can be seen that if $\eta^{2}+\gamma^{2}<1$, three eigenvalues of $D$ are negative, i.e., $D$ is a negativedefinite matrix. Clearly, $\lambda_{2}$ is the maximum eigenvalue of $D$. This implies that

$$
\begin{aligned}
& \left.\frac{d}{d t} V(t, \phi)\right|_{(1)} \\
& \quad \leq\left(-1+\sqrt{\eta^{2}+\gamma^{2}}\right)\left(\delta(t)\|x(t)\|^{2}+(1-\alpha) \lambda_{\min }(G)\|x(t-\tau(t))\|^{2}+\rho(t)|f(\sigma(t))|^{2}\right) \\
& \quad \leq\left(-1+\sqrt{\eta^{2}+\gamma^{2}}\right)\left(\delta\|x(t)\|^{2}+\rho|f(\sigma(t))|^{2}\right) .
\end{aligned}
$$

Since $\sigma(t) f(\sigma(t)) \geq k_{1} \sigma^{2}(t)$, we have $|f(\sigma(t))| \geq k_{1}|\sigma(t)|$. Thus,

$$
\begin{aligned}
& \left.\frac{d}{d t} V(t, \phi)\right|_{(1)} \\
& \leq\left(-1+\sqrt{\eta^{2}+\gamma^{2}}\right)\left(\delta\|x(t)\|^{2}+\rho{k_{1}}^{2} \sigma^{2}(t)\right) \\
& \leq\left(-1+\sqrt{\eta^{2}+\gamma^{2}}\right) \min \left(\delta, \rho k_{1}{ }^{2}\right)\left\|\left[\begin{array}{c}
x(t) \\
\sigma(t)
\end{array}\right]\right\|^{2}
\end{aligned}
$$

This shows that, as to all $f \in F_{\left[k_{1}, k_{2}\right]},\left.\frac{d}{d t} V(t, \phi)\right|_{(1)}$ is negative definite. Based on Lyapunov's theorem, system (1) is absolutely stable, which completes the proof of Theorem 1.

Because asymptotical stability is a property of the trajectories of a system as time tends to infinity, we just need to ensure that the above requirements can be met when time $t$ is sufficiently large. Therefore, A2 and A3 can be rewritten as follows. There exists $T \geq 0$ such that when $t>T$, the corresponding conditions hold. Particularly, A3 can be rewritten as a new form of the upper limit, that is, the following A4 is valid.

A4: It is assumed that

$$
\varlimsup_{t \rightarrow \infty} \frac{\|P B(t)\|}{\sqrt{\delta(t)(1-\alpha) \lambda_{\min }(G)}}=\bar{\eta}, \quad \varlimsup_{t \rightarrow \infty} \frac{\left\|P b(t)+\frac{1}{2} c(t)\right\|}{\sqrt{\delta(t) \rho(t)}}=\bar{\gamma}
$$

where $\bar{\eta}, \bar{\gamma}$ are constants.

The following corollaries are more convenient in practical situations.

Corollary 1 Under A1, A2 and A4, if the inequality

$$
\bar{\eta}^{2}+\bar{\gamma}^{2}<1
$$

holds, then system (1) is absolutely stable.

Proof According to the property of the upper limit, if A4 holds, for any $\varepsilon>0$, there exists $T(T \geq 0)$ such that when $t>T$ the following hold:

$$
\frac{\|P B(t)\|}{\sqrt{\delta(t)(1-\alpha) \lambda_{\min }(G)}} \leq \bar{\eta}+\varepsilon, \quad \frac{\left\|P b(t)+\frac{1}{2} c(t)\right\|}{\sqrt{\delta(t) \rho(t)}} \leq \bar{\gamma}+\varepsilon .
$$

Let

$$
\eta=\bar{\eta}+\varepsilon, \quad \gamma=\bar{\gamma}+\varepsilon,
$$


then inequality (2) in Theorem 1 holds when $t>T$. By Theorem 1 , if there exists $\varepsilon>0$ such that

$$
\psi(\varepsilon)=(\bar{\eta}+\varepsilon)^{2}+(\bar{\gamma}+\varepsilon)^{2}<1,
$$

then system (1) is absolutely stable. We notice that the known condition $\psi(0)=\bar{\eta}^{2}+\bar{\gamma}^{2}<1$, and $\psi(\varepsilon)$ is a continuous function of $\varepsilon$, thus a positive real number $\varepsilon$ which is sufficiently small can be found such that $\psi(\varepsilon)<1$. This completes the proof of Corollary 1 .

In fact, if we define $\delta=1-\left(\bar{\eta}^{2}+\bar{\gamma}^{2}\right)$ and take $\varepsilon=\frac{-(\bar{\eta}+\bar{\gamma})+\sqrt{(\bar{\eta}+\bar{\gamma})^{2}+\delta}}{2}$, then we have $\varepsilon>0$ and $(\bar{\eta}+\varepsilon)^{2}+(\bar{\gamma}+\varepsilon)^{2}=1-\frac{\delta}{2}<1$.

Corollary 2 Under A1, A2 and A4, if the inequality

$$
\bar{\eta}+\bar{\gamma}<1
$$

holds, then system (1) is absolutely stable.

Proof From $\bar{\eta} \geq 0, \bar{\gamma} \geq 0$, obviously, we have

$$
\bar{\eta}^{2}+\bar{\gamma}^{2} \leq(\bar{\eta}+\bar{\gamma})^{2}
$$

If $\bar{\eta}+\bar{\gamma}<1$, i.e. , $(\bar{\eta}+\bar{\gamma})^{2}<1$, then inequality (3) is valid. Thus, Corollary 2 holds by Corollary 1.

Particulary, if the coefficients of system (1) are bounded, the above conclusions are still accurate. Certainly, the above criteria are also true for Lurie systems with constant coefficients.

\section{Absolute stability of Lurie systems with multiple nonlinearities}

Consider the following time-varying delay Lurie indirect control system with variable coefficients and multiple nonlinearities:

$$
\left\{\begin{array}{l}
\dot{x}(t)=A(t) x(t)+B(t) x(t-\tau(t))+\sum_{j=1}^{m} b_{j}(t) f_{j}\left(\sigma_{j}(t)\right), \\
\dot{\sigma}_{i}(t)=c_{i}^{T}(t) x(t)-\rho_{i}(t) f_{i}\left(\sigma_{i}(t)\right) \quad(i=1,2, \ldots, m), \\
x(t)=\varphi(t), \quad t \in[-h, 0],
\end{array}\right.
$$

where $x(t) \in R^{n} ; \sigma_{i}(t) \in R(i=1,2, \ldots, m) ; A(t), B(t)$ are $n \times n$ matrices; $b_{i}(t), c_{i}(t)(i=$ $1,2, \ldots, m)$ are $n$-dimensional column vectors; $\tau(t)$ is time delay; $\rho_{i}(t) \geq \rho_{i}>0(i=$ $1,2, \ldots, m), \rho_{i}$ are constants. $A(t), B(t), b_{i}(t), c_{i}(t), \rho_{i}(t)$ are continuous in $[0, \infty) . \varphi(t)$ is the initial condition. The nonlinearities $f_{i}(\cdot)(i=1,2, \ldots, m)$ are continuous and satisfy the sector condition:

$$
F_{\left[k_{i 1}, k_{i 2}\right]}=\left\{f_{i}(\cdot) \mid f_{i}(0)=0 ; k_{i 1} \sigma_{i}^{2}(t) \leq \sigma_{i}(t) f_{i}\left(\sigma_{i}(t)\right) \leq k_{i 2} \sigma_{i}^{2}(t), \sigma_{i}(t) \in R-\{0\}\right\},
$$

where $k_{i 1}, k_{i 2}$ are given constants satisfying $k_{i 2}>k_{i 1}>0$.

Definition 2 System (4) is said to be absolutely stable if its zero solution is globally asymptotically stable for any nonlinearity $f_{i}(\cdot) \in F_{\left[k_{i 1}, k_{i 2}\right]}(i=1,2, \ldots, m)$. 
In addition to $\mathrm{A} 1$ and $\mathrm{A} 2$, the following assumptions are needed for system (4).

A5: For any $t \in[0, \infty)$, assume that

$$
\frac{\|P B(t)\|}{\sqrt{\delta(t)(1-\alpha) \lambda_{\min }(G)}} \leq \eta, \quad \frac{\left\|P b_{j}(t)+\frac{1}{2} c_{j}(t)\right\|}{\sqrt{\delta(t) \rho_{j}(t)}} \leq \gamma_{j}
$$

where $\eta, \gamma_{j}(j=1,2, \ldots, m)$ are constants.

Theorem 2 Under A1, A2 and A5, if the inequality

$$
\eta^{2}+\sum_{i=1}^{m} \gamma_{i}^{2}<1
$$

holds, then system (4) is absolutely stable.

Proof Using matrices $P$ and $G$, a Lyapunov-Krasovskii functional candidate can be chosen as

$$
V(t, \phi)=x^{T}(t) P x(t)+\int_{t-\tau(t)}^{t} x^{T}(s) G x(s) d s+\sum_{i=1}^{m} \int_{0}^{\sigma_{i}(t)} f_{i}(s) d s
$$

where $\phi(\theta)=\left[x^{T}(t+\theta) \sigma_{1}(t) \cdots \sigma_{m}(t)\right]^{T}, \theta \in[-h, 0], t \geq 0$. Similarly to the proof of Theorem 1, it can be verified that $V(t, \phi)$ satisfies the conditions required by Lyapunov's theorem.

Next calculating the time derivative of $V(t, \phi)$ along the trajectories of system (4) yields

$$
\begin{aligned}
\frac{d}{d t} V(t, \phi) & \left.\right|_{(4)} \\
= & 2 x^{T}(t) P \dot{x}(t)+x^{T}(t) G x(t) \\
& -(1-\dot{\tau}(t)) x^{T}(t-\tau(t)) G x(t-\tau(t))+\sum_{i=1}^{m} f_{i}\left(\sigma_{i}(t)\right) \dot{\sigma}_{i}(t) \\
= & 2 x^{T}(t) P\left[A(t) x(t)+B(t) x(t-\tau(t))+\sum_{j=1}^{m} b_{j}(t) f_{j}\left(\sigma_{j}(t)\right)\right] \\
& +x^{T}(t) G x(t)-(1-\dot{\tau}(t)) x^{T}(t-\tau(t)) G x(t-\tau(t)) \\
& +\sum_{i=1}^{m} f_{i}\left(\sigma_{i}(t)\right)\left(c_{i}^{T}(t) x(t)-\rho_{i}(t) f_{i}\left(\sigma_{i}(t)\right)\right) \\
= & x^{T}(t)\left[P A(t)+A^{T}(t) P+G\right] x(t)+2 x^{T}(t) P B(t) x(t-\tau(t)) \\
& +2 x^{T}(t) P \sum_{j=1}^{m} b_{j}(t) f_{j}\left(\sigma_{j}(t)\right)-(1-\dot{\tau}(t)) x^{T}(t-\tau(t)) G x(t-\tau(t)) \\
& +\sum_{i=1}^{m} f_{i}\left(\sigma_{i}(t)\right) c_{i}^{T}(t) x(t)-\sum_{i=1}^{m} \rho_{i}(t) f_{i}^{2}\left(\sigma_{i}(t)\right) .
\end{aligned}
$$


Likewise, in the light of A1, A2 and the property of norm, the following will be obtained:

$$
\begin{aligned}
&\left.\frac{d}{d t} V(t, \phi)\right|_{(4)} \\
& \leq- \delta(t)\|x(t)\|^{2}+2\|P B(t)\|\|x(t)\|\|x(t-\tau(t))\| \\
&+2 \sum_{j=1}^{m}\left\|P b_{j}(t)+\frac{1}{2} c_{j}(t)\right\|\|x(t)\|\left|f_{j}\left(\sigma_{j}(t)\right)\right| \\
& \quad-(1-\alpha) \lambda_{\min }(G)\|x(t-\tau(t))\|^{2}-\sum_{i=1}^{m} \rho_{i}(t) f_{i}^{2}\left(\sigma_{i}(t)\right) .
\end{aligned}
$$

In order to take advantage of A5 and the unbounded terms in the coefficients of system (4), let us take $\sqrt{\delta(t)}\|x(t)\|, \sqrt{(1-\alpha) \lambda_{\min }(G)}\|x(t-\tau(t))\|$ and $\sqrt{\rho_{i}(t)}\left|f_{i}\left(\sigma_{i}(t)\right)\right|(i=1,2, \ldots, m)$ as the following variables of the quadratic form. Further estimating the right-hand side of $\left.\frac{d}{d t} V(t, \phi)\right|_{(4)}$ based on A5 yields

$$
\begin{aligned}
\left.\frac{d}{d t} V(t, \phi)\right|_{(4)} & \\
\leq & \delta(t)\|x(t)\|^{2} \\
& +\frac{2\|P B(t)\|}{\sqrt{\delta(t)(1-\alpha) \lambda_{\min }(G)}}[\sqrt{\delta(t)}\|x(t)\|] \cdot\left[\sqrt{(1-\alpha) \lambda_{\min }(G)}\|x(t-\tau(t))\|\right] \\
& +2 \sum_{j=1}^{m} \frac{\left\|P b_{j}(t)+\frac{1}{2} c_{j}(t)\right\|}{\sqrt{\delta(t) \rho_{j}(t)}}[\sqrt{\delta(t)}\|x(t)\|] \cdot\left[\sqrt{\rho_{j}(t)}\left|f_{j}\left(\sigma_{j}(t)\right)\right|\right] \\
& -(1-\alpha) \lambda_{\min }(G)\|x(t-\tau(t))\|^{2}-\sum_{i=1}^{m} \rho_{i}(t) f_{i}^{2}\left(\sigma_{i}(t)\right) \\
\leq & -\delta(t)\|x(t)\|^{2}+2 \eta[\sqrt{\delta(t)}\|x(t)\|] \cdot\left[\sqrt{\left.(1-\alpha) \lambda_{\min }(G)\|x(t-\tau(t))\|\right]}\right. \\
& +2 \sum_{j=1}^{m} \gamma_{j}[\sqrt{\delta(t)}\|x(t)\|] \cdot\left[\sqrt{\rho_{j}(t)}\left|f_{j}\left(\sigma_{j}(t)\right)\right|\right] \\
& -(1-\alpha) \lambda_{\min }(G)\|x(t-\tau(t))\|^{2}-\sum_{i=1}^{m} \rho_{i}(t) f_{i}^{2}\left(\sigma_{i}(t)\right) .
\end{aligned}
$$

Rewriting the right-hand side of the above inequality, it follows that

$$
\begin{aligned}
\left.\frac{d}{d t} V(t, \phi)\right|_{(4)} \leq & {\left[\begin{array}{c}
\sqrt{\delta(t)}\|x(t)\| \\
\sqrt{(1-\alpha) \lambda_{\min }(G)}\|x(t-\tau(t))\| \\
\sqrt{\rho_{1}(t)}\left|f_{1}\left(\sigma_{1}(t)\right)\right| \\
\vdots \\
\sqrt{\rho_{m}(t)}\left|f_{m}\left(\sigma_{m}(t)\right)\right|
\end{array}\right]^{T} } \\
& \times D\left[\begin{array}{c}
\sqrt{\delta(t)}\|x(t)\| \\
\sqrt{(1-\alpha) \lambda_{\min }(G)}\|x(t-\tau(t))\| \\
\sqrt{\rho_{1}(t)}\left|f_{1}\left(\sigma_{1}(t)\right)\right| \\
\vdots \\
\sqrt{\rho_{m}(t)}\left|f_{m}\left(\sigma_{m}(t)\right)\right|
\end{array}\right],
\end{aligned}
$$


where

$$
D=\left[\begin{array}{ccccc}
-1 & \eta & \gamma_{1} & \cdots & \gamma_{m} \\
\eta & -1 & 0 & \cdots & 0 \\
\gamma_{1} & 0 & -1 & \cdots & 0 \\
\cdots & \cdots & \cdots & \cdots & \cdots \\
\gamma_{m} & 0 & 0 & \cdots & -1
\end{array}\right] .
$$

In the following section we will prove that the right-hand side of (5) is a negative-definite function. Firstly, let us show that matrix $D$ is negative definite. Calculating the characteristic polynomial of $D$ yields

$$
\begin{aligned}
& |\lambda I-D| \\
& =\left|\begin{array}{ccccc}
\lambda+1 & -\eta & -\gamma_{1} & \cdots & -\gamma_{m} \\
-\eta & \lambda+1 & 0 & \cdots & 0 \\
-\gamma_{1} & 0 & \lambda+1 & \cdots & 0 \\
\cdots & \cdots & \cdots & \cdots & \cdots \\
-\gamma_{m} & 0 & 0 & \cdots & \lambda+1
\end{array}\right| \\
& =(\lambda+1)^{m}\left[(\lambda+1)^{2}-\left(\eta^{2}+\sum_{i=1}^{m} \gamma_{i}^{2}\right)\right] .
\end{aligned}
$$

It can easily be seen that $\lambda=-1$ is an eigenvalue of multiplicity $m$, and the other two eigenvalues are given by $\lambda=-1 \pm \sqrt{\eta^{2}+\sum_{i=1}^{m} \gamma_{i}^{2}}$. Therefore, if $\eta^{2}+\sum_{i=1}^{m} \gamma_{i}^{2}<1$, all eigenvalues of $D$ are negative, i.e., $D$ is negative definite.

Let us denote the largest eigenvalue of $D$ by $\beta$, namely, $\beta=-1+\sqrt{\eta^{2}+\sum_{i=1}^{m} \gamma_{i}^{2}}$. From (6), the following will be obtained:

$$
\begin{aligned}
& \left.\frac{d}{d t} V(t, \phi)\right|_{(4)} \\
& \quad \leq \beta\left(\delta(t)\|x(t)\|^{2}+(1-\alpha) \lambda_{\min }(G)\|x(t-\tau(t))\|^{2}+\sum_{i=1}^{m} \rho_{i}(t)\left|f_{i}\left(\sigma_{i}(t)\right)\right|^{2}\right) \\
& \quad \leq \beta\left(\delta\|x(t)\|^{2}+\sum_{i=1}^{m} \rho_{i}\left|f_{i}\left(\sigma_{i}(t)\right)\right|^{2}\right) .
\end{aligned}
$$

Since $\sigma_{i}(t) f_{i}\left(\sigma_{i}(t)\right) \geq k_{i 1} \sigma_{i}^{2}(t)$, then $\left|f_{i}\left(\sigma_{i}(t)\right)\right| \geq k_{i 1}\left|\sigma_{i}(t)\right|(i=1,2, \ldots, m)$ holds. Therefore, from the above inequality, we obtain

$$
\begin{aligned}
& \left.\frac{d}{d t} V(t, \phi)\right|_{(4)} \\
& \quad \leq \beta\left(\delta\|x(t)\|^{2}+\sum_{i=1}^{m} \rho_{i} k_{i 1}^{2} \sigma_{i}^{2}(t)\right) \\
& \quad \leq \beta \min \left(\delta, \rho_{1} k_{11}^{2}, \ldots, \rho_{m} k_{m 1}^{2}\right)\left\|\left[\begin{array}{c}
x(t) \\
\sigma_{1}(t) \\
\vdots \\
\sigma_{m}(t)
\end{array}\right]\right\|^{2} .
\end{aligned}
$$


Because $\beta<0$, for any nonlinearity $f_{i}(\cdot)$ satisfying the given sector condition, we get $\left.\frac{d}{d t} V(t, \phi)\right|_{(4)}$ is negative definite. Thus, system (4) is absolutely stable by Lyapunov's theorem. This completes the proof of Theorem 2.

Similarly to the case of single nonlinearity, in order to guarantee that system (4) is absolutely stable, A5 in Theorem 2 can be rewritten as follows: There exists $T \geq 0$ such that when $t>T$ the corresponding conditions hold. Therefore, $\eta, \gamma_{j}(j=1,2, \ldots, m)$ in A5 can be calculated by the upper limit (if the corresponding upper limit is a finite value).

A6: It is assumed that

$$
\varlimsup_{t \rightarrow \infty} \frac{\|P B(t)\|}{\sqrt{\delta(t)(1-\alpha) \lambda_{\min }(G)}}=\bar{\eta}, \quad \varlimsup_{t \rightarrow \infty} \frac{\left\|P b_{j}(t)+\frac{1}{2} c_{j}(t)\right\|}{\sqrt{\delta(t) \rho_{j}(t)}}=\bar{\gamma}_{j},
$$

where $\bar{\eta}, \bar{\gamma}_{j}(j=1,2, \ldots, m)$ are constants.

Corollary 3 Under A1, A2 and A6, if the inequality

$$
\bar{\eta}^{2}+\sum_{i=1}^{m} \bar{\gamma}_{i}^{2}<1
$$

holds, then system (4) is absolutely stable.

The proof follows similar steps as in the proof of Corollary 1 , and thus is omitted here. According to Corollary 3, it is easy to obtain the following Corollary 4.

Corollary 4 Under A1, A2 and A6, if the inequality

$$
\bar{\eta}+\sum_{j=1}^{m} \bar{\gamma}_{j}<1
$$

holds, then system (4) is absolutely stable.

\section{Numerical simulations}

In this section, the validity of the proposed approach will be shown by numerical examples.

Example 1 Consider the time-varying delay Lurie indirect control system with variable coefficients and single nonlinearity

$$
\left\{\begin{aligned}
& {\left[\begin{array}{l}
\dot{x}_{1}(t) \\
\dot{x}_{2}(t)
\end{array}\right]=} {\left[\begin{array}{cc}
-2 t-\frac{1}{2} & 1 \\
t & -3 t-\frac{1}{2}
\end{array}\right]\left[\begin{array}{l}
x_{1}(t) \\
x_{2}(t)
\end{array}\right] } \\
&+\left[\begin{array}{cc}
\sqrt{\frac{t}{2}} & 0 \\
0 & \sqrt{\frac{t}{6}}
\end{array}\right]\left[\begin{array}{l}
x_{1}(t-\tau(t)) \\
x_{2}(t-\tau(t))
\end{array}\right]+\left[\begin{array}{c}
-\frac{1}{2} t \\
0
\end{array}\right] f(\sigma(t)), \\
& \dot{\sigma}(t)=\left[\begin{array}{ll}
t & \sqrt{t}
\end{array}\right]\left[\begin{array}{l}
x_{1}(t) \\
x_{2}(t)
\end{array}\right]-(t+1) f(\sigma(t))
\end{aligned}\right.
$$

where $\tau(t)=3+0.5 \sin t, f(\cdot) \in F_{[0.01,100]}$. 
In comparison with system (1), the coefficient matrices are as follows:

$$
\begin{array}{ll}
A(t) & =\left[\begin{array}{cc}
-2 t-\frac{1}{2} & 1 \\
t & -3 t-\frac{1}{2}
\end{array}\right], \quad B(t)=\left[\begin{array}{cc}
\sqrt{\frac{t}{2}} & 0 \\
0 & \sqrt{\frac{t}{6}}
\end{array}\right], \quad b(t)=\left[\begin{array}{c}
-\frac{1}{2} t \\
0
\end{array}\right], \\
c(t) & =\left[\begin{array}{c}
t \\
\sqrt{t}
\end{array}\right], \quad \rho(t)=t+1 .
\end{array}
$$

Now let us verify that this system satisfies all the conditions of Theorem 1 .

Firstly, it is obvious that $0 \leq \tau(t) \leq 3.5=h, \dot{\tau}(t)=0.5 \cos t \leq 0.5<1$. We have $\alpha=0.5$. Thus, A1 is satisfied.

Then, let $P=G=I$, it follows that

$$
P A(t)+A^{T}(t) P+G=\left[\begin{array}{cc}
-4 t & t+1 \\
t+1 & -6 t
\end{array}\right] .
$$

It is easy to obtain

$$
\lambda\left(P A(t)+A^{T}(t) P+G\right) \leq-5 t+\sqrt{2 t^{2}+2 t+1} .
$$

Furthermore, let $T=1.5$, when $t>T$, we have

$$
\lambda\left(P A(t)+A^{T}(t) P+G\right)<-5 t+\sqrt{2}(t+1)=-(5-\sqrt{2}) t+\sqrt{2}<-(4-\sqrt{2}) t .
$$

Thus, we can choose

$$
\delta(t)=(4-\sqrt{2}) t
$$

Note that if $t>T$, we have

$$
-\delta(t) \leq-\xi=-(4 \sqrt{2}-2)
$$

Thus, A2 is satisfied. In addition,

$$
\begin{aligned}
& \frac{\|P B(t)\|}{\sqrt{\delta(t)(1-\alpha) \lambda_{\min }(G)}}=\frac{1}{\sqrt{4-\sqrt{2}}}<\frac{1}{\sqrt{2}} \\
& \frac{\left\|P b(t)+\frac{1}{2} c(t)\right\|}{\sqrt{\delta(t) \rho(t)}}=\frac{\sqrt{t} / 2}{\sqrt{(4-\sqrt{2}) t \cdot(t+1)}} \leq \frac{1}{\sqrt{t} \cdot \sqrt{16-4 \sqrt{2}}}<\frac{1}{\sqrt{15}} .
\end{aligned}
$$

Hence, $\eta=\frac{1}{\sqrt{2}}, \gamma=\frac{1}{\sqrt{15}}$, that is, A3 is satisfied.

It is clear that $\eta^{2}+\gamma^{2}=\frac{17}{30}<1$. Summarising the conditions obtained, we conclude that Theorem 1 is applicable and system (6) is absolutely stable. In order to carry out a numerical simulation, let

$$
f(\sigma(t))=2 \sigma(t)+\sin \sigma(t)
$$


Now it can be proved that $f(\sigma(t))$ belongs to $F_{[0.01,100]}$. Obviously, $f(0)=0$. Thus, we just need to show that if $\sigma(t) \neq 0$, the following inequalities

$$
0.01 \sigma^{2}(t) \leq \sigma(t)[2 \sigma(t)+\sin \sigma(t)] \leq 100 \sigma^{2}(t)
$$

i.e.,

$$
0.01 \leq 2+\frac{\sin \sigma(t)}{\sigma(t)} \leq 100
$$

are valid.

First we know, if $0<|\sigma(t)|<\frac{\pi}{2}$, we have

$$
\cos \sigma(t)<\frac{\sin \sigma(t)}{\sigma(t)}<1
$$

Hence,

$$
\begin{aligned}
& 2+\frac{\sin \sigma(t)}{\sigma(t)}<2+1=3<100, \\
& 2+\frac{\sin \sigma(t)}{\sigma(t)}>2+\cos \sigma(t)>2-1=1>0.01 .
\end{aligned}
$$

Thus, in such a case, (7) hold.

$$
\text { If }|\sigma(t)| \geq \frac{\pi}{2} \text {, because of }|\sin \sigma(t)| \leq 1 \text {, we have }
$$

$$
2+\frac{\sin \sigma(t)}{\sigma(t)} \leq 2+\frac{|\sin \sigma(t)|}{|\sigma(t)|} \leq 2+\frac{1}{|\sigma(t)|} \leq 2+\frac{1}{\pi / 2}<100
$$

that is, the right-hand side of (7) is valid. Moreover,

$$
2+\frac{\sin \sigma(t)}{\sigma(t)} \geq 2-\frac{|\sin \sigma(t)|}{|\sigma(t)|} \geq 2-\frac{1}{|\sigma(t)|} \geq 2-\frac{2}{\pi} \geq 2-1=1>0.01
$$

that is, the left-hand side of $(7)$ is valid. Thus, $f(\sigma(t)) \in F_{[0.01,100]}$.

The numerical simulation is carried out by Matlab. Suppose the initial condition is $\left[x_{1}(t) x_{2}(t) \sigma(0)\right]^{T}=\left[\begin{array}{lll}1 & 1 & 0\end{array}\right]^{T}, t \in[-h, 0]$. The state response of system (6) is shown in Figure 1 .

It can be seen from Figure 1 that the zero solution of system (6) is asymptotically stable. Changing the form of $f(\sigma(t))$ and carrying out a corresponding numerical simulation demonstrate that system (6) is asymptotically stable as long as $f(\cdot) \in F_{[0.01,100]}$. Thus, it is absolutely stable. This example illustrates that the simulation result is in perfect accordance with theoretical conclusions.

Furthermore, in this paper, the derived theorems and corollaries are sufficient conditions. This implies that system (1) may be still asymptotically stable although some conditions are not satisfied. For this example, let $f(\sigma(t))=\sigma^{2}(t)$, and the rest of the parameters remain unchanged. Although $f(\sigma(t))$ does not belong to any $F_{\left[k_{1}, k_{2}\right]}$, it is found that sys- 


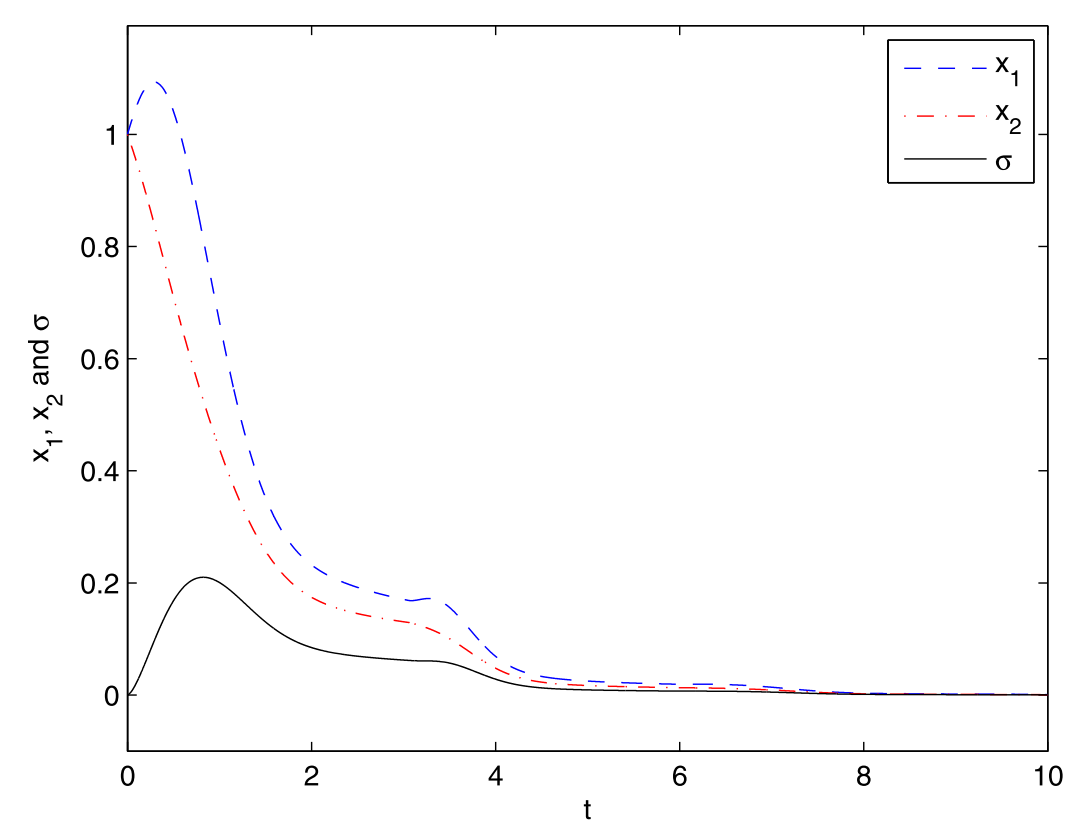

Figure 1 The state response of system (6) (with $f(\sigma(t))=2 \sigma(t)+\sin \sigma(t))$.

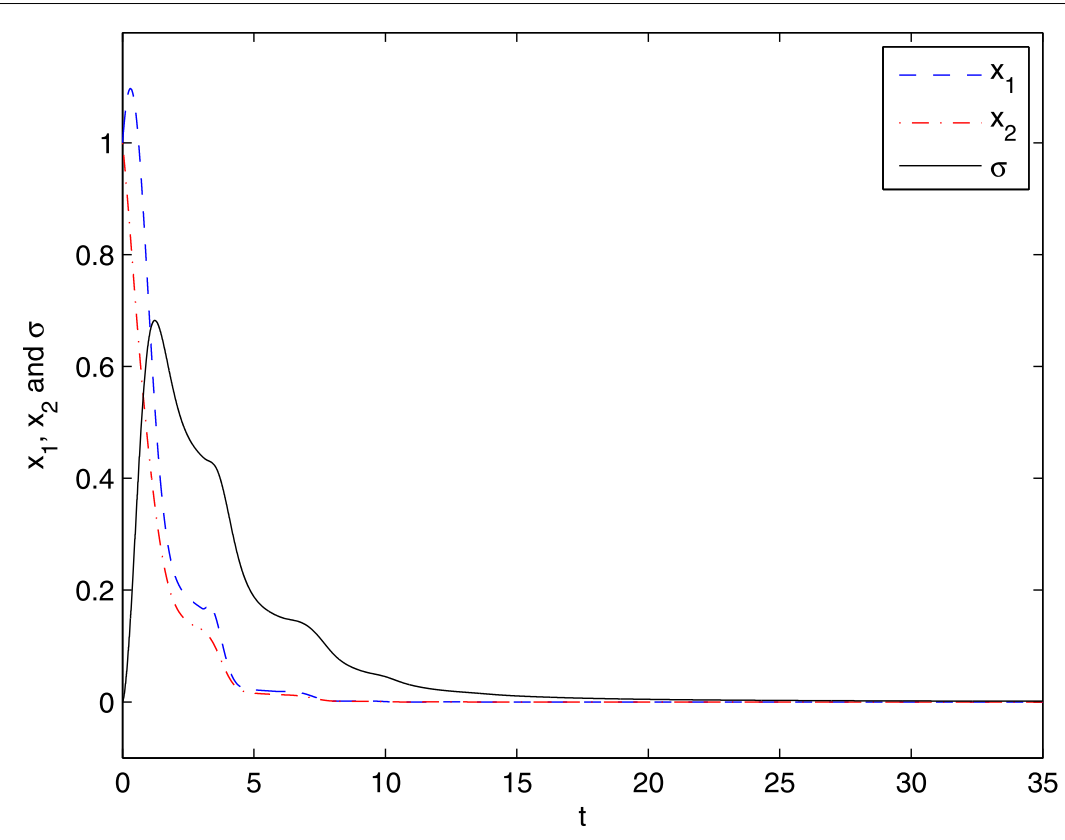

Figure 2 The state response of system (6) (with $\left.f(\sigma(t))=\sigma^{2}(t)\right)$.

tem (6) is still asymptotically stable by simulation, as shown in Figure 2. Therefore, it is possible to extend the absolute stability region of parameters for system (1). This will be explored in our future works.

The above selected $\tau(t)$ is derivable everywhere. Next, $\tau(t)$ is rewritten as a continuous and piecewise differentiable function. 


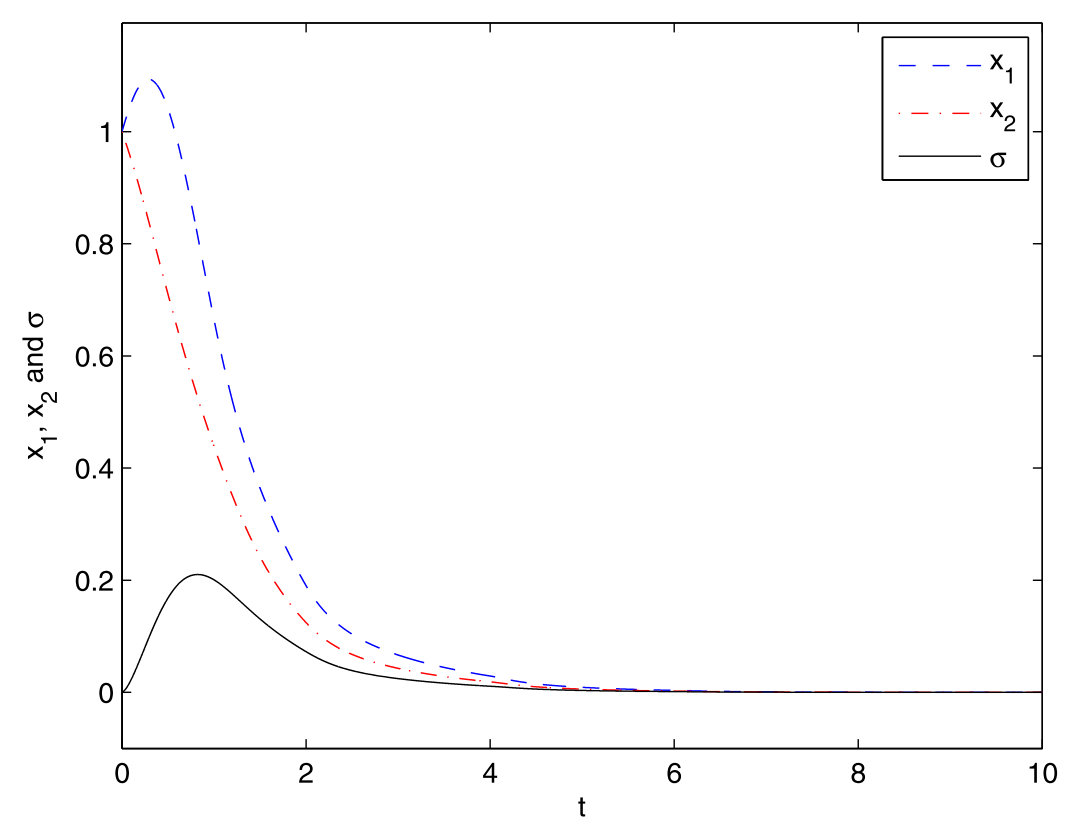

Figure 3 The state response of the system in Example 2.

Example 2 We still consider system (6), the time delay is given by

$$
\tau(t)= \begin{cases}1, & t<2 \\ 0.5 t, & 2 \leq t \leq 4 \\ 2, & t>4\end{cases}
$$

The other parameters remain unchanged. Here $\tau(t) \leq 2$ means $h=2$. Note that $\tau(t)$ is not derivable at $t=2$ and $t=4$, but it has right and left derivative. Combined with A1, we have $\dot{\tau}(t) \leq 0.5$. Thus, $\alpha=0.5$. Similarly to Example 1 , this system is absolutely stable. By utilising Matlab, the simulation result is shown in Figure 3.

It is worth noting that the coefficients $A(t), B(t), b(t), c(t), \rho(t)$ in Example 1 and Example 2 are unbounded. This is the novelty of the paper. All theorems and corollaries are suitable for systems whose coefficient matrices are unbounded. Actually, for Lurie systems with bounded or constant coefficients, all results are also true. Now an example of Lurie system with constant coefficients is presented.

Example 3 Consider the time-varying delay Lurie indirect control system with constant coefficients

$$
\left\{\begin{aligned}
& {\left[\begin{array}{l}
\dot{x}_{1}(t) \\
\dot{x}_{2}(t)
\end{array}\right]=} {\left[\begin{array}{cc}
-1.1 & 0.2 \\
0.1 & -1
\end{array}\right]\left[\begin{array}{l}
x_{1}(t) \\
x_{2}(t)
\end{array}\right]+\left[\begin{array}{ll}
0.3 & 0.3 \\
0.3 & 0.2
\end{array}\right]\left[\begin{array}{l}
x_{1}(t-\tau(t)) \\
x_{2}(t-\tau(t))
\end{array}\right] } \\
&+\left[\begin{array}{l}
1 \\
1
\end{array}\right] f(\sigma(t)), \\
& \dot{\sigma}(t)=\left[\begin{array}{ll}
-1 & -1
\end{array}\right]\left[\begin{array}{l}
x_{1}(t) \\
x_{2}(t)
\end{array}\right]-10 f(\sigma(t)),
\end{aligned}\right.
$$


where $\tau(t)=3+0.5 \sin t, f(\cdot) \in F_{[0.01,100]}$. Here,

$$
\begin{aligned}
& A(t)=\left[\begin{array}{cc}
-1.1 & 0.2 \\
0.1 & -1
\end{array}\right], \quad B(t)=\left[\begin{array}{ll}
0.3 & 0.3 \\
0.3 & 0.2
\end{array}\right], \\
& b(t)=\left[\begin{array}{l}
1 \\
1
\end{array}\right], \quad c(t)=\left[\begin{array}{l}
-1 \\
-1
\end{array}\right], \quad \rho(t)=10
\end{aligned}
$$

are all constant matrices or constants.

Now we verify that this system satisfies all the conditions of Theorem 1 .

First, it is obvious that $0 \leq \tau(t) \leq 3.5=h, \dot{\tau}(t)=0.5 \cos t \leq 0.5<1$. We have $\alpha=0.5$.

Thus, A1 is satisfied. Then let $P=G=I$, it follows that

$$
P A(t)+A^{T}(t) P+G=\left[\begin{array}{cc}
-1.2 & 0.3 \\
0.3 & -1
\end{array}\right] .
$$

It is easy to obtain

$$
\lambda\left(P A(t)+A^{T}(t) P+G\right) \leq-1.1+\sqrt{0.1} .
$$

Thus, we have

$$
\xi=\delta(t)=1.1-\sqrt{0.1}
$$

Then, A2 is satisfied. In addition,

$$
\begin{aligned}
& \frac{\|P B(t)\|}{\sqrt{\delta(t)(1-\alpha) \lambda_{\min }(G)}}=\frac{\sqrt{0.155+\sqrt{0.023125}}}{\sqrt{0.5(1.1-\sqrt{0.1})}}<0.9, \\
& \frac{\left\|P b(t)+\frac{1}{2} c(t)\right\|}{\sqrt{\delta(t) \rho(t)}}=\frac{\sqrt{0.5}}{\sqrt{10(1.1-\sqrt{0.1})}}<0.3 .
\end{aligned}
$$

Hence, we have $\eta=0.9, \gamma=0.3$ in A3.

It is clear that $\eta^{2}+\gamma^{2}=0.9<1$, which means that the conditions of Theorem 1 are satisfied. The conclusion could be made that system (8) is absolutely stable. Let

$$
f(\sigma(t))=2 \sigma(t)+\sin \sigma(t)
$$

Suppose the initial condition is $\left[x_{1}(t) x_{2}(t) \sigma(0)\right]^{T}=\left[\begin{array}{lll}1 & 1 & 0\end{array}\right]^{T}, t \in[-h, 0]$. The simulation result is obtained using Matlab, as shown in Figure 4.

Figure 4 indicates that the zero solution of system (8) is asymptotically stable. This verifies theoretical results. Changing $f(\sigma(t))$ to simulate yields that system (8) is asymptotically stable so long as $f(\cdot) \in F_{[0.01,100]}$, i.e., system (8) is absolutely stable. Thus, the results in this paper are true for Lurie systems with constant coefficients.

Next, an example of Lurie system with multiple nonlinearities is introduced. 


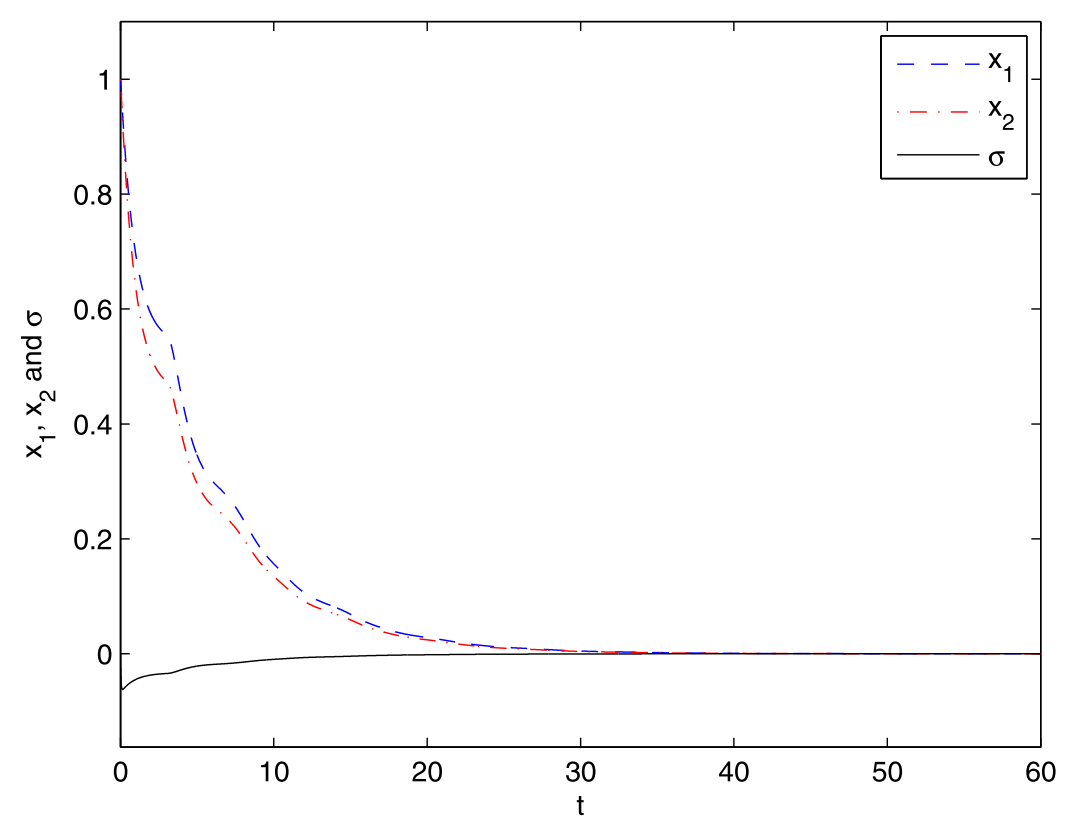

Figure 4 The state response of system (8).

Example 4 Consider the time-varying delay Lurie indirect control system with variable coefficients and two nonlinearities

$$
\left\{\begin{array}{l}
\dot{x}(t)=A(t) x(t)+B(t) x(t-\tau(t))+\sum_{i=1}^{2} b_{i}(t) f_{i}\left(\sigma_{i}(t)\right), \\
\dot{\sigma}_{i}(t)=c_{i}^{T}(t) x(t)-\rho_{i}(t) f_{i}\left(\sigma_{i}(t)\right) \quad(i=1,2)
\end{array}\right.
$$

where $\tau(t)=3+0.5 \sin t, f_{i}(\cdot) \in F_{[0.01,100]}, i=1,2$ and

$$
\begin{array}{ll}
A(t) & =\left[\begin{array}{cc}
-3 t-\frac{1}{2} & t \\
1 & -4 t-\frac{1}{2}
\end{array}\right], \quad B(t)=\left[\begin{array}{cc}
\sqrt{\frac{t}{4}} & 0 \\
0 & \sqrt{\frac{t}{6}}
\end{array}\right], \\
b_{1}(t)=\left[\begin{array}{c}
\sqrt{t} \\
t
\end{array}\right], & b_{2}(t)=\left[\begin{array}{c}
-t \\
2 t
\end{array}\right], \\
c_{1}(t)=\left[\begin{array}{c}
1 \\
-2 t
\end{array}\right], & c_{2}(t)=\left[\begin{array}{c}
2 t \\
-4 t
\end{array}\right], \\
\rho_{1}(t)=t+1, & \rho_{2}(t)=2 t+1 .
\end{array}
$$

Now we verify that this system satisfies all the conditions of Corollary 4.

Firstly, it is obvious that $0 \leq \tau(t) \leq 3.5=h, \dot{\tau}(t)=0.5 \cos t \leq 0.5<1$. We know that $\alpha=$ 0.5. Thus A1 is satisfied.

Then let $P=G=I$, it follows that

$$
P A(t)+A^{T}(t) P+G=\left[\begin{array}{cc}
-6 t & t+1 \\
t+1 & -8 t
\end{array}\right] .
$$


It is easy to obtain

$$
\lambda\left(P A(t)+A^{T}(t) P+G\right) \leq-7 t+\sqrt{2 t^{2}+2 t+1} .
$$

Further, let $T=2$. Then, when $t>T$, we have

$$
\lambda\left(P A(t)+A^{T}(t) P+G\right)<-5 t<-10 .
$$

Thus A2 is satisfied with $\delta(t)=5 t, \xi=-10$. In addition,

$$
\begin{aligned}
& \lim _{t \rightarrow \infty} \frac{\|P B(t)\|}{\sqrt{\delta(t)(1-\alpha) \lambda_{\min }(G)}}=\lim _{t \rightarrow \infty} \frac{\sqrt{t}}{2 \sqrt{5 t \cdot 0.5}}=\frac{1}{\sqrt{10}}, \\
& \lim _{t \rightarrow \infty} \frac{\left\|P b_{1}(t)+\frac{1}{2} c_{1}(t)\right\|}{\sqrt{\delta(t) \rho_{1}(t)}}=\lim _{t \rightarrow \infty} \frac{0.5+\sqrt{t}}{\sqrt{5 t(t+1)}}=0, \\
& \lim _{t \rightarrow \infty} \frac{\left\|P b_{2}(t)+\frac{1}{2} c_{2}(t)\right\|}{\sqrt{\delta(t) \rho_{2}(t)}}=0 .
\end{aligned}
$$

We recall the fact that the upper limit always exists if the limit exists, and it is equal to the limit value. Hence, for A6 we have $\bar{\eta}=\frac{1}{\sqrt{10}}, \bar{\gamma}_{1}=\bar{\gamma}_{2}=0$.

It is clear that $\bar{\eta}+\bar{\gamma}_{1}+\bar{\gamma}_{2}=\frac{1}{\sqrt{10}}<1$. Thus, all the conditions in Corollary 4 are satisfied, that is, system (9) is absolutely stable.

In order to carry out the numerical simulation, let

$$
f_{1}(\sigma(t))=2 \sigma(t)+\sin \sigma(t), \quad f_{2}(\sigma(t))= \begin{cases}\sigma(t), & |\sigma(t)|<1, \\ \sigma^{3}(t), & 1 \leq|\sigma(t)| \leq 2 \\ 4 \sigma(t), & |\sigma(t)|>2\end{cases}
$$

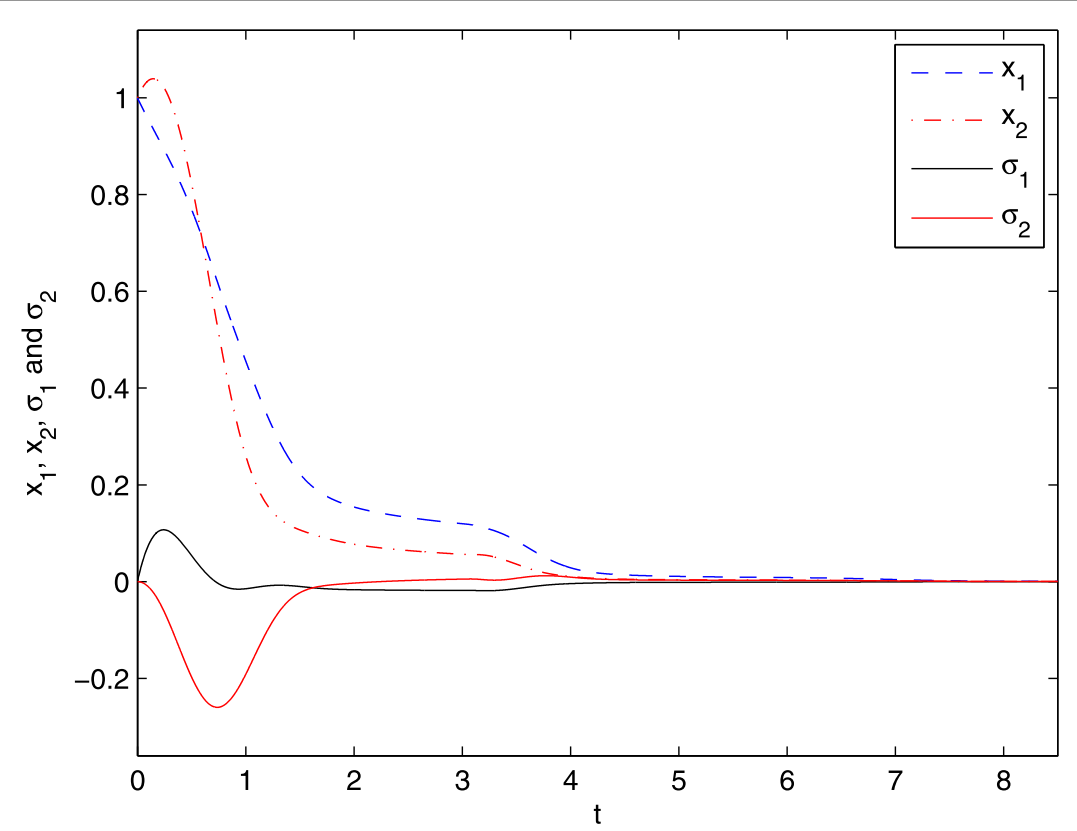

Figure 5 The state response of system (9). 
Suppose the initial condition of the system is given by

$$
\left[\begin{array}{llll}
x_{1}(t) & x_{2}(t) & \sigma_{1}(0) & \sigma_{2}(0)
\end{array}\right]^{T}=\left[\begin{array}{llll}
1 & 1 & 0 & 0
\end{array}\right]^{T}, \quad t \in[-h, 0] .
$$

With the aid of Matlab, the state response of system (9) is shown in Figure 5. It illustrates that the numerical simulation result is completely consistent with the theoretical conclusion.

\section{Conclusion}

The absolute stability problem of time-varying delay Lurie indirect control systems with variable coefficients has been investigated in this paper. Based on Lyapunov stability theory, some sufficient conditions and several simple and practical corollaries have been obtained. The results in this paper are especially applicable to checking the absolute stability of time-varying delay Lurie indirect control systems with unbounded coefficients. The validity of the proposed criteria has been demonstrated by numerical examples.

Competing interests

The authors declare that they have no competing interests.

Authors' contributions

The authors have made the same contribution. All authors read and approved the final manuscript.

\section{Author details}

'School of Mathematics and Physics, University of Science and Technology Beijing, Beijing, 100083, China. ${ }^{2}$ Leeds Sustainability Institute, Leeds Beckett University, Leeds, LS2 9EN, UK.

\section{Acknowledgements}

This work was supported by the National Natural Science Foundation of China (grant number 61174209).

Received: 16 August 2016 Accepted: 18 January 2017 Published online: 03 February 2017

\section{References}

1. Lur'e, Al, Postnikov, VN: On the theory of stability of control system. Prikl. Mat. Meh. 8(3), 283-286 (1944)

2. Liberzon, MR: Essays on the absolute stability theory. Autom. Remote Control 67(10), 1610-1644 (2006)

3. Gelig, AH, Leonov, GA, Fradkov, AL: Nonlinear Systems. Frequency and Matrix Inequalities. Fizmatlit, Moscow (2008)

4. Lur'e, Al: Some Nonlinear Problems in the Theory of Automatic Control. H. M. Stationery Office, London (1957)

5. Aizerman, MA, Gantmaher, FR: Absolute Stability of Regulator Systems. Holden-Day, San Francisco (1964)

6. Xie, H: Theories and Applications of Absolute Stability. Science Press, Beijing (1986)

7. Khusainov, DY, Shatyrko, AV: Absolute stability of multi-delay regulation systems. J. Autom. Inf. Sci. 27(3), 33-42 (1995)

8. Chen, W-H, Guan, Z-H, Lu, X-M: Absolute stability of Lurie indirect control systems with multiple variable delays. Acta Math. Sin. 47(6), 1063-1070 (2004)

9. Gan, Z-X, Ge, W-G: Absolute stability of a class of multiple nonlinear Lurie control systems with delay. Acta Math. Sin. 43(4), 633-638 (2000)

10. Tian, J, Zhong, S, Xiong, L: Delay-dependent absolute stability of Lurie control systems with multiple time-delays. Appl. Math. Comput. 188(1), 379-384 (2007)

11. Cao, J, Zhong, S: New delay-dependent condition for absolute stability of Lurie control systems with multiple time-delays and nonlinearities. Appl. Math. Comput. 194(1), 250-258 (2007)

12. Nam, PT, Pathirana, PN: Improvement on delay dependent absolute stability of Lurie control systems with multiple time delays. Appl. Math. Comput. 216(3), 1024-1027 (2010)

13. Daryoush, BS, Soheila, DC: Improvement on delay dependent absolute stability of Lurie control systems with multiple time-delays and nonlinearities. World J. Model. Simul. 10(1), 20-26 (2014)

14. Shatyrko, A, Diblík, J, Khusainov, D, Růžičková, M: Stabilization of Lur'e-type nonlinear control systems by Lyapunov-Krasovskii functionals. Adv. Differ. Equ. 2012, 229 (2012)

15. Wang, T-C, Wang, Y-C, Hong, L-R: Absolute stability for Lurie control system with unbound time delays. J. China Univ. Min. Technol. 14(1), 67-69 (2005)

16. Zeng, H-B, He, Y, Wu, M, Feng, Z-Y: New absolute stability criteria for Lurie nonlinear systems with time-varying delay. Control Decis. 25(3), 346-350 (2010)

17. Liu, P-L: Delayed decomposition approach to the robust absolute stability of a Lur'e control system with time-varying delay. Appl. Math. Model. 40(3), 2333-2345 (2016)

18. Shatyrko, AV, Khusainov, DY: Absolute interval stability of indirect regulating systems of neutral type. J. Autom. Inf. Sci. 42(6), 43-54 (2010)

19. Shatyrko, AV, Khusainov, DY, Diblik, J, Baštinec, J, Rivolova, A: Estimates of perturbation of nonlinear indirect interval control system of neutral type. J. Autom. Inf. Sci. 43(1), 13-28 (2011) 
20. Shatyrko, A, van Nooijen, RRP, Kolechkina, A, Khusainov, D: Stabilization of neutral-type indirect control systems to absolute stability state. Adv. Differ. Equ. 2015, 64 (2015)

21. Liao, F-C, Li, A-G, Sun, F-B: Absolute stability of Lurie systems and Lurie large-scale systems with multiple operators and unbounded coefficients. J. Univ. Sci. Technol. B 31(11), 1472-1479 (2009)

22. Wang, D, Liao, F: Absolute stability of Lurie direct control systems with time-varying coefficients and multiple nonlinearities. Appl. Math. Comput. 219(9), 4465-4473 (2013)

23. Liao, F, Wang, D: Absolute stability criteria for large-scale Lurie direct control systems with time-varying coefficients. Sci. World J. 2014, Article ID 631604 (2014). doi:10.1155/2014/631604

24. Burton, TA: Uniform asymptotical stability in functional differential equations. Proc. Am. Math. Soc. 68(2), 195-199 (1978)

25. Burton, TA: Stability and Periodic Solutions of Ordinary and Functional Differential Equations. Academic Press, New York (1985)

26. Lefschetz, S: Stability of Nonlinear Control Systems. Academic Press, New York (1965)

Submit your manuscript to a SpringerOpen ${ }^{\circ}$ journal and benefit from:

- Convenient online submission

Rigorous peer review

- Immediate publication on acceptance

- Open access: articles freely available online

- High visibility within the field

- Retaining the copyright to your article 